BMJ Open

Sport \&

Exercise

Medicine

\title{
A systematic approach to the characterisation of human impact injury scenarios in sport
}

\author{
Thomas Payne, ${ }^{1}$ Séan Mitchell, ${ }^{1}$ Ben Halkon, ${ }^{1}$ Richard Bibb ${ }^{2}$
}

To cite: Payne T, Mitchell S, Halkon B, et al. A systematic approach to the characterisation of human impact injury scenarios in sport. BMJ Open Sport Exerc Med 2015;2:e000017. doi:10.1136/bmjsem-2015000017

- Prepublication history for this paper is available online To view these files please visit the journal online (http://dx.doi.org/10.1136/ bmjsem-2015-000017).

Accepted 18 August 2015

\section{CrossMark}

\footnotetext{
${ }^{1}$ Sports Technology Institute, Loughborough University, Loughborough, UK ${ }^{2}$ Design School, Loughborough University, Loughborough, UK
}

Correspondence to Dr Séan Mitchell; s.r.mitchell@|boro.ac.uk

\section{ABSTRACT}

Background: In contact sports (eg, American football or rugby), injuries resulting from impacts are widespread. There have been several attempts to identify and collate, within a conceptual framework, factors influencing the likelihood of an injury. To effectively define an injury event it is necessary to systematically consider all potential causal factors but none of the previous approaches are complete in this respect.

Aims: First, to develop a superior deterministic contextual sequential (DCS) model to promote a complete and logical description of interrelated injury event factors. Second, to demonstrate systematic use of the model to construct enhanced perspectives for impact-injury research.

Method: Previous models were examined and elements of best practice synthesised into a new DCS framework description categorising the types of causal factors influencing injury. The approach's internal robustness is demonstrated by consideration of its completeness, lack of redundancy and logical consistency.

Results: The model's external validity and worth are demonstrated through its use to generate superior descriptive injury models, experimental protocols and intervention opportunities. Comprehensive research perspectives have been developed using a common rugby impact-injury scenario as an example; this includes: a detailed description of the injury event, an experimental protocol for a human-on-surrogate reconstruction, and a series of practical interventions in the sport of rugby aimed at mitigating the risk of injury.

Conclusions: Our improved characterisation tool presents a structured approach to identify pertinent factors relating to an injury.

\section{INTRODUCTION Background}

In contact sports, injuries sustained from impacts are common and debilitating and place a significant financial burden on society in terms of productive societal days lost. A study by Schmikli et $a l^{1}$ conducted in the Netherlands estimated the indirect cost

\section{What this study adds?}

- A comprehensive model and list of influencing factors that can be used to accurately describe sports impact injuries.

- A framework that facilitates characterisation and understanding of specific sports injuries illustrated with specific examples.

- A structured method of developing experimental protocol to study sports injuries and intervention opportunities in a format that promotes consideration of many key factors.

of this work absence from physical activity related injury to be $\$ 525$ million annually. There are many complex interacting factors that can influence the likelihood of injury occurrence, which can arise from behavioural, strategic, biomechanical or medical incitements. Each dynamic loading event produces a series of distinct injury outcomes that can be further differentiated by factors such as 'situational environment', exposure level and performance of personal protective equipment. ${ }^{2}$ There may also be conflicting factors which adversely affect injury prevention. For example, Hagel and Meeuwisse observed that athletes adopted a risk compensation attitude when they were given superior protective equipment, and typically performed impacts with a greater intensity and recklessness as they perceived themselves to be safer. Overall, it is likely that a combination of several factors will result in an injury. ${ }^{4}$ To reduce the risk of injuries it is important to develop a thorough understanding of the influencing factors, their interaction and the sequence of events preceding an injury.

\section{Previous approaches}

There have been several attempts to describe a conceptual framework for the relationship between factors determining an injury event. These models are typically 
deterministic and can be categorised by three distinct approaches:

- A risk accumulation and intensification model (ie, injury occurs because of the accumulation of risk factors making the injury outcome increasingly probable).

- A mechanical phenomena sequence (ie, injury occurs due to a sequence of interrelated mechanical loading events).

- An event sequence entity matrix (ie, the Haddon matrix).

The first risk accumulation and intensification model was named the "multifactorial model'. ${ }^{5}$ It is based on the principal that multiple causal factors contribute towards a single injury outcome (figure 1). It is considered that the likelihood of injury is dependent on internal risk factors (eg, biomechanics, conditioning of the athlete), exposure to external risk factors (eg, equipment, weather) and an inciting event (eg, contact).

This model has since been adapted, notably by Bahr and Krosshaug ${ }^{6}$ who identified specific risk factors influencing an anterior cruciate ligament (ACL) injury. This model substantiated the multifactorial model framework and applied it to aid in the identification of the aetiology of a specific sports injury scenario. Another significant adaptation to the model was introduced by Meeuwisse $^{7}$ who proposed a dynamic causal injury model which considered a reflective change in risk, related to previous impacts altering the predisposition of athletes to injuries. A similar approach was also undertaken by Gissane $e t$ al. ${ }^{8}$

The mechanical phenomena sequence was first presented by Wismans et at and named the 'biomechanical dependency chain' (figure 2). It highlights the causal series of events from initial contact to injury occurrence. The general model suggests that when a body is subjected to external loading it deforms and in doing so triggers a biomechanical response from the body which varies within and between people. If the body deforms beyond a recoverable limit, the injury tolerance level will be exceeded, leading to a specific injury which is the function of the injury mechanism, resulting in damage to anatomical structures and impairment. This model has since been adapted by McIntosh, ${ }^{10}$ who presented a similar core structure with a specific focus towards the

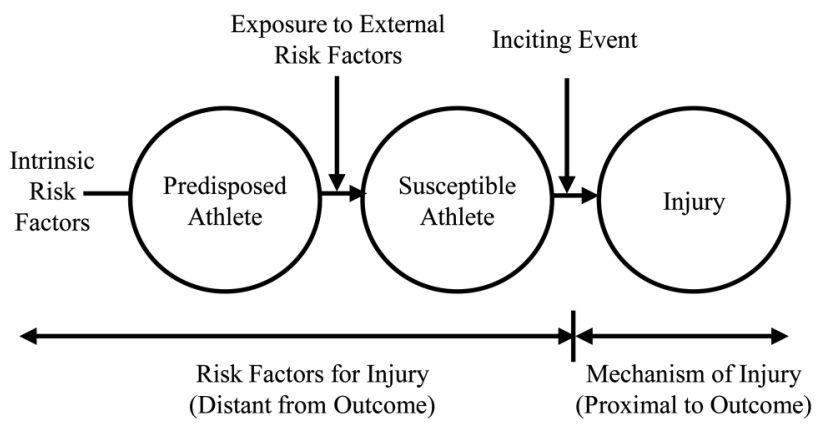

Figure 1 Multifactorial Model. ${ }^{5}$

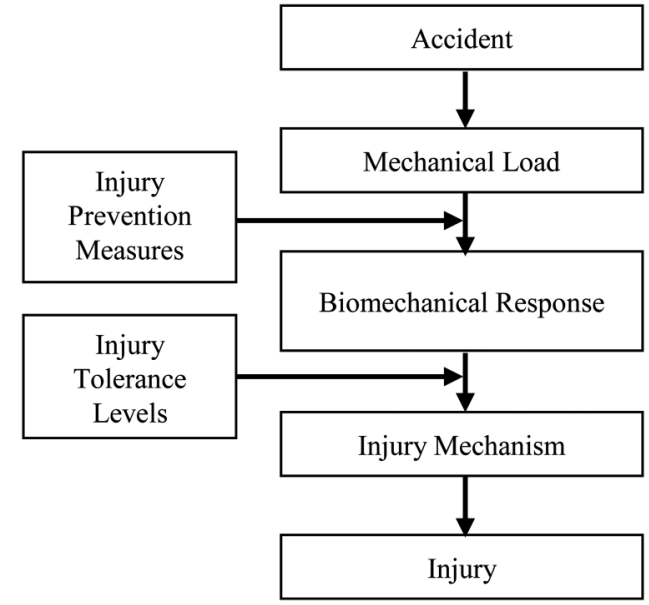

Figure 2 Load-injury Model. ${ }^{9}$

effects of training interventions and psychological developments on mitigating injury risk. The model expanded the general approach using elements from the multifactorial model to identify many external factors that influence injury risk and provided an indication of potential areas for interventions.

The Haddon ${ }^{11}$ matrix is a cross-tabulation of important elements relating to injury, presented in a nine-cell matrix. It provides the opportunity to offer a preventative strategy in each cell corresponding to the relevant headings. This simple chart was initially designed to analyse injury prevention methods in the automotive industry. The structure of the matrix ensures systematic consideration is given to a range of known pertinent factors and can reveal areas in which knowledge is lacking. The matrix has also been presented in a sports context, notably by Bahr and Mæhlum ${ }^{12}$ who suggested the use of the model to conceptualise risk factors with a given injury problem using the headings: human, equipment and environment. This has since been applied by authors to describe injuries in American football ${ }^{13}$ and association football. ${ }^{14}$

Each of these models presents a different method for describing the injury event but none of them ensure all factors are given full consideration. The risk accumulation and intensification model does not describe in sufficient detail the deterministic series of events preceding injury and fails to address the nature of the body's response to impact. The mechanical phenomena sequence does not adequately differentiate between sequential event types. The point of application for intervention methods (ie, injury prevention measures) is also too narrow and does not consider opportunities to mitigate risk at other stages (figure 2). In addition, both of these model types are conceptual and somewhat academic; they suggest a method of describing injuries but do not clearly enable efficient execution of tasks dependent on this description. While the nine-cell Haddon matrix provides useful structure, it leaves much still to do with little inspiration. The absence of a list of 
influencing factors also makes it possible to overlook elements of importance.

\section{STUDY AIMS}

The principal aims of this study pertain to: (1) the development of a new conceptual impact-injury framework to systematically provide a more complete description of the causal factors relating to a particular injury event; and (2) application of the model to show its potential uses in scenario definition, experimental protocol design and identification of intervention opportunities.

\section{IMPACT-INJURY MODEL \\ Core model}

To better understand the causes of injuries it is necessary to consider the potential causal factors at each stage preceding it. A contextual example of the framework has been provided in reverse from injury occurrence to context. It is believed that injury occurs when human tissue experiences an overload condition. This condition is dependent on several factors which can be arranged in a dependency chain. For example, the overload occurrence in a particular structure will depend on the tissue type (eg, muscle) and preconditioning physiological factors (eg, fatigue); overloading will concern a particular tissue response (eg, strain) to a load transfer mechanism (eg, tensile stress) imposed by a load condition (eg, impact) and associated resistance (eg, inertia); the loading condition will depend on the type of incident (eg, human on human impact) resulting from a particular sport (eg, Rugby).

The determinative contextual sequential (DCS) model uses a deterministic flow diagram at its core with a dependency chain reflecting the deterministic relationships between the injury factors arising from elements relating to context, physiology and impact mechanics. This model, presented in figure 3, maps the path from the participant performing the sports activity to the injury occurrence. The framework consists of a dependency chain using diagrammatic elements from traditional flow chart construction. The elements start at a general contextual level and become more specific as the particular injury becomes more defined.

\section{Influencing factors}

The framework provided by figure 3 enables systematic identification, classification and ordering of all factors that may influence any sports injury event.

An attempt has been made to exhaustively list without redundancy the factors relating to each of the elements in the deterministic framework (table 1), that is, the conditions dictating the outcome. Each set of factors presents a basis to characterise the individual components of the injury event and is intrinsically linked to the previous series. For example, if the 'sports incident' involves a football stud impact, the 'loading factors' will consider factors such as the geometry and stiffness of the stud. The framework attempts to provide a clear succession of exhaustible factors that aims to completely describe the phenomena occurring in the injury event.

'These factors were derived both from a collation of noted influencing factors from previous conceptual frameworks studies ${ }^{5-7} 1015$ and mechanical injury epidemiology and classification literature. ${ }^{16-18}$ While this can never provide a completely exhaustive description of every factor influencing an injury event, it is sufficiently detailed to stimulate useful consideration of many important aspects.'

\section{APPLICATIONS}

\section{Scenario definition}

The DCS framework provides a valuable tool in describing specific impact injury scenarios. The comprehensive set of influencing factors promotes consideration of many key issues associated with the particular impact event. This level of prompted detail is absent from in all previous models and would likely result in a partial description of the factors relating to the injury.

Using the model, a given injury can be investigated retrospectively to generate a detailed description of the causal factors relating to a specific sports injury scenario. For most effective usage of the model the scenario in which the injury occurred must be well defined (eg, rugby shoulder tackle to the anterior thigh).

Examining the sports specific injury scenario in more detail, at a contextual level, the parameters in which the sport is played must be defined (eg, contact sport (1.1), 15 players per team (1.6), typically played on grass surfaces (1.2)). The scenario then becomes more defined, identifying factors relating to the specific incident (eg, human-human impact (2.1), deliberate contact (2.2), $50 \mathrm{~mm}$ thickness foam padding worn on impacting players shoulder (2.1)).

This scenario can then be used to determine a specific set of loading factors describing the manner in which the impactor interfaces with the target body (eg, ball carrier travelling at $7 \mathrm{~m} / \mathrm{s}$, impacted by defensive player travelling at $3 \mathrm{~m} / \mathrm{s}$ in frontal impact (3.1)). The specific load transfer factors from this impact type may concern, for example, a $100 \mathrm{~kg}, 1.80 \mathrm{~m}$ tall target player braced
Figure 3 Deterministic contextual sequential Core Framework.

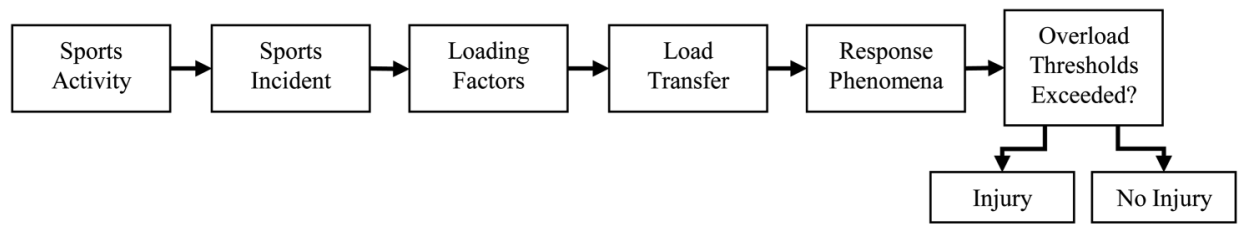


Table 1 DCS influencing factors checklist

1. Sports activity

2. Sports incident

3. Loading factors

\section{Load transfer factors}

5. Response phenomena

6. Overload thresholds exceeded?

7. Injury
1.1. Game rules (contact/no contact)

1.2. Equipment (goals, projectiles, apparel, implements and surfaces)

1.3. Player exposure

1.4. Gender propensity (mixed/single sex)

1.5. Team/individual

1.6. Popularity (no. of participants, frequency of play)

1.7. Attitude/behaviour

1.8. Tactics

1.9. Temperature and humidity

2.1. Contact type: human (with or without personal protective equipment) versus:

- Human (with or without personal protective equipment)

- Projectile

- Surface

- Obstacles (goals, nets, posts, boards. etc)

- Implements (bats, sticks, etc)

2.2. Deliberate (active) or accidental (passive) contact

2.3. Two or more (striker \& target(s))

2.4. Constraints (striker \& target): free, driven and planted

2.5. Impact location-segment detail

3.1. Relative incoming velocity (target and striker)

3.2. Relative surface alignment (target and striker)

3.3. Specific surface geometry (target and striker)

3.4. Surface properties (target and striker); external friction, lubrication

3.5. Effective inertia of striker (mass, moment of inertia and distribution)

3.6. Striker stiffness (inertial resistance)

3.7. Degrees of freedom/constraints (pivot friction, moments, secondary inertia)

4.1. Effective inertia of target (mass, moment of inertia and mass distribution)

4.2. Anthropometrics

4.3. Material characteristics:

- Type (bone, muscle etc.)

- State (tensed/relaxed/flexed/extended)

- Mechanical properties (immediate surface and affected structures)

4.4. Spatial geometry (physical location of material)

4.5. Target degrees of freedom (at each of the joints; rotational and translational degrees)

4.6. Internal friction and lubrication

4.7. Ground reaction forces

5.1. Impact duration

5.2. Tissue displacement

5.3. Segment velocity (target and striker)

5.4. Joint displacement

5.5. Acceleration

5.6. Shock

5.7. Vibration

5.8. Physiological factors (eg, muscle tension, tendon reflex, localised pressure change)

5.9. Temperature change

6.1. Compressive stresses and strains

6.2. Tensile stresses and strains

6.3. Shear stresses and strains

6.4. Cyclic fatigue

6.5. Subject specific property variation (eg, age, physical condition, pre-existing damage or deformity)

6.6. Multiaxial stress (resulting in yield or failure of tissue)

7.1. Muscle contusions

7.2. Bone fractures

7.3. Joint dislocations

7.4. Lacerations (compression, tearing of body tissue by blunt impact)

7.5. Blistering (friction, rubbing)

7.6. Incisions (cutting by sharp implement)

7.7. Puncture (penetrating with sharp implement)

7.8. Avulsions (forceful tearing of body tissue)

7.9. Sprains/strains

7.10. Nerve entrapment

7.11. Organ failure

7.12. Concussion and soft tissue head injuries 
for impact (4.1, 4.2 and 4.3). The response phenomena then consider the specific mechanical loading occurring to the tissues (eg, compression of skin, subcutaneous adipose and muscle tissues (5.2); global recoil velocity of thigh segment (5.3)).

The manner in which these response phenomena are experienced by the human are then considered, identifying whether they exceed the specific tissue overload tolerances for the particular human (eg, magnitudes of compressive (6.1) and tensile (6.2) stresses and strains on tissues experienced relative to particular tissue injury thresholds (6.5)). If the particular tissue tolerance is exceeded it may result in a particular injury outcome (eg, muscle contusions (7.1), ligaments sprains (7.9)).

These human injury tolerances, particularly those related to soft tissues, are very dynamic and vary greatly within and between people dependant on a number of factors such as physical condition, injury site, impact orientation, rate of impact and levels of muscle contraction. These factors are particularly pertinent to soft tissue injuries. There are, however, some practical guidelines that could be usefully followed. For example, bone fracture is suggested to occur under axial compressive loads greater than $8900 \mathrm{~N},{ }^{19}$ while muscle contusions are suggested to occur when mechanical contact pressures greater than $1 \mathrm{MPa}$ occur. $^{20}$

\section{Experimental protocol development}

Another key application of the model is in experimental protocol development. The core framework and influencing factors model can be either used to identify elements where there is insufficient prior knowledge to perform an experiment (eg, unknown effective mass of striker) or used to examine human response to approximated loading conditions. Using the DCS model, these elements can then be assessed in greater detail through prioritising and simplifying key experimental factors to ensure a controlled and cost-effective experiment. This level of consideration is not explicitly accounted for in other models and they do not directly facilitate these applications in injury biomechanics research.

Experimental protocols can be in the form of: laboratory reconstructions, human gameplay scenarios or virtual simulations. The DCS model can be used as a checklist for factors to approximate, control and measure, assessing each individual element sequentially to define a complete list of parameters. These elements have been divided in a cross-tabulation to provide a framework from which each element can be categorically assessed with the column headings: human, equipment and environment.

Approximations are factors which can or must be simplified to make testing feasible. It is important to identify fully which factors are being approximated, the reasons for their approximation and the likely outcomes of the decisions made. For example, considering a laboratory human-on-surrogate reconstruction of the Rugby tackle scenario, at a contextual level, the clothing worn and sports pitch would be factors that need approximating, while the material properties of the target ball carriers thigh may require approximation in a synthetic surrogate. Controls are factors not relevant to the particular experiment that must be prevented from interfering. For example, temperature and humidity of the laboratory, contact type and location should be controlled in this scenario. Measures are the dependent and independent variables from the experiment. In this scenario, measures can range from player questionnaires to get a better understanding of the types of injury scenarios commonly sustained in the sport to measurement of the kinematics experienced in the impact.

This approach has been successfully exploited in laboratory reconstructions of basketball ${ }^{21}$ and American football $^{22}$ impacts to determine the kinetics and kinematics involved. Figure 4 shows an example of a human-on-surrogate impact laboratory reconstruction with annotations showing a subset of approximations, controls and measures from the study.

The experimental protocol model has similarly been applied in computational optimisation studies. Using the model, mechanical approximations of a cricket ball and human knee impact scenario were defined and used to evaluate different human surrogate material simulants in a sports impact surrogate. ${ }^{23}$

\section{Interventions}

The DCS model can also be exploited to systematically identify intervention opportunities that can be used to mitigate the risk of injury. Similar to the experimental protocol development, a cross-tabulation has been used to categorise these potential opportunities and ensure completeness is maintained. Heading categories: human, equipment and environment were selected as distinct entities that would invoke greater consideration to possible opportunities (figure 5).

With a systematic and comprehensive framework the key role of the intervention model is to expose the opportunities for intervention, to classify existing approaches and stimulate creative formulation of new approaches. Three main intervention methods were typically considered to be: risk elimination, where the risk is removed entirely; risk modification, aimed at changing the system and reducing the potential severity; and consequence mitigation, aimed at protecting the individual after the injury has occurred.

A practical example of an impact-injury scenario in which a series of interventions have been applied has been in cervical spine injuries sustained in Rugby Union scrimmaging. At a contextual level, legislation has been employed to prevent participants playing in competition when they are not physically ready. In 1984, IRB legislation was changed to make the game safer and since further empirical research based law modifications have been enforced. ${ }^{24}$ This includes but is not limited to: restricting competition where there is a mismatch of skills or strengths in forward players in youth 


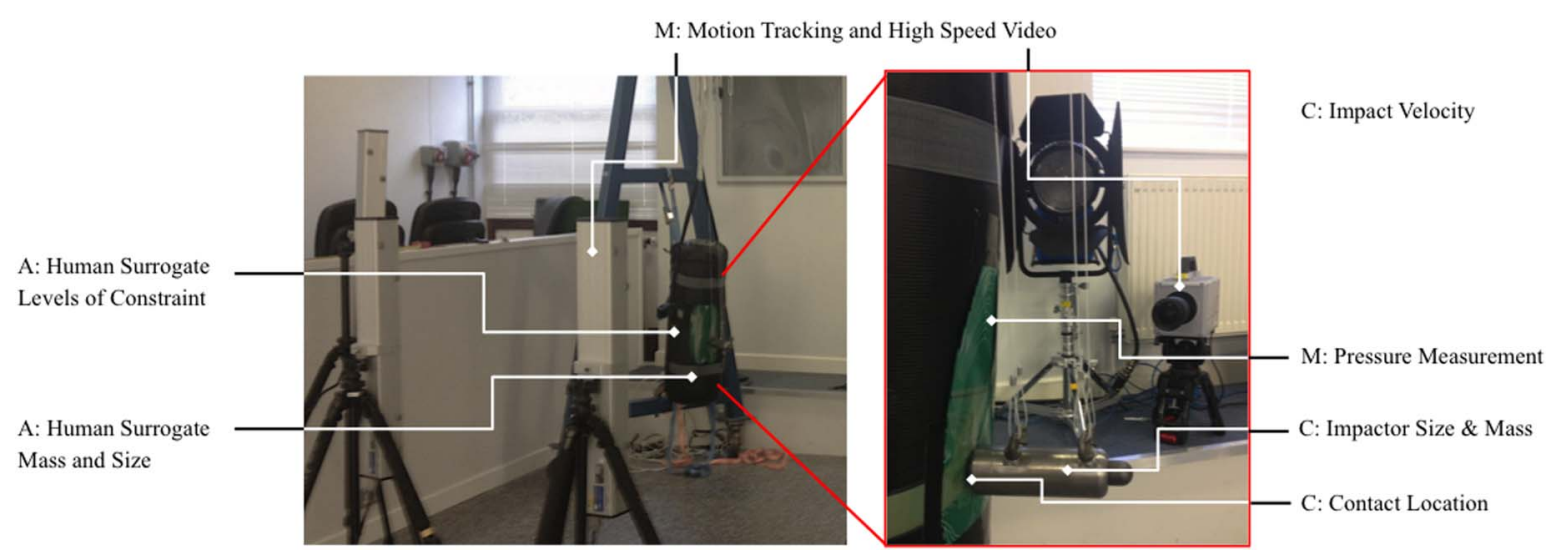

Figure 4 Basketball impact laboratory reconstruction (A, Approximation; C, Control; M, Measure) (Adapted from Halkon et a ${ }^{2}$ ).

competition and monitoring the physical condition of professional players following injury. ${ }^{25}$

Considering loading factors, in New Zealand, a RugbySmart education programme has been introduced to inform coaches, referees and players of ways to reduce the risk of injury in games. ${ }^{26}$ This has also been applied in the formation of a series of neck-injury prevention programmes to inform coaches. ${ }^{27}$ Studies have also investigated the forces generated in rugby scrums ${ }^{28} 29$ and consequently a new prebind engagement protocol has been recommended to limit the severity of initial compressive impacts. ${ }^{30}$ Furthermore interventions relating to response phenomena have been investigated, a study by Naish et $a l^{31}$ recommended that front-row forward players undertake neck strengthening exercises to mitigate the risk of cervical spine injury.

\section{DISCUSSION}

\section{Appraisal of conceptual model}

The DCS model provides a toolkit from which to investigate factors pertaining to a specific injury event. It

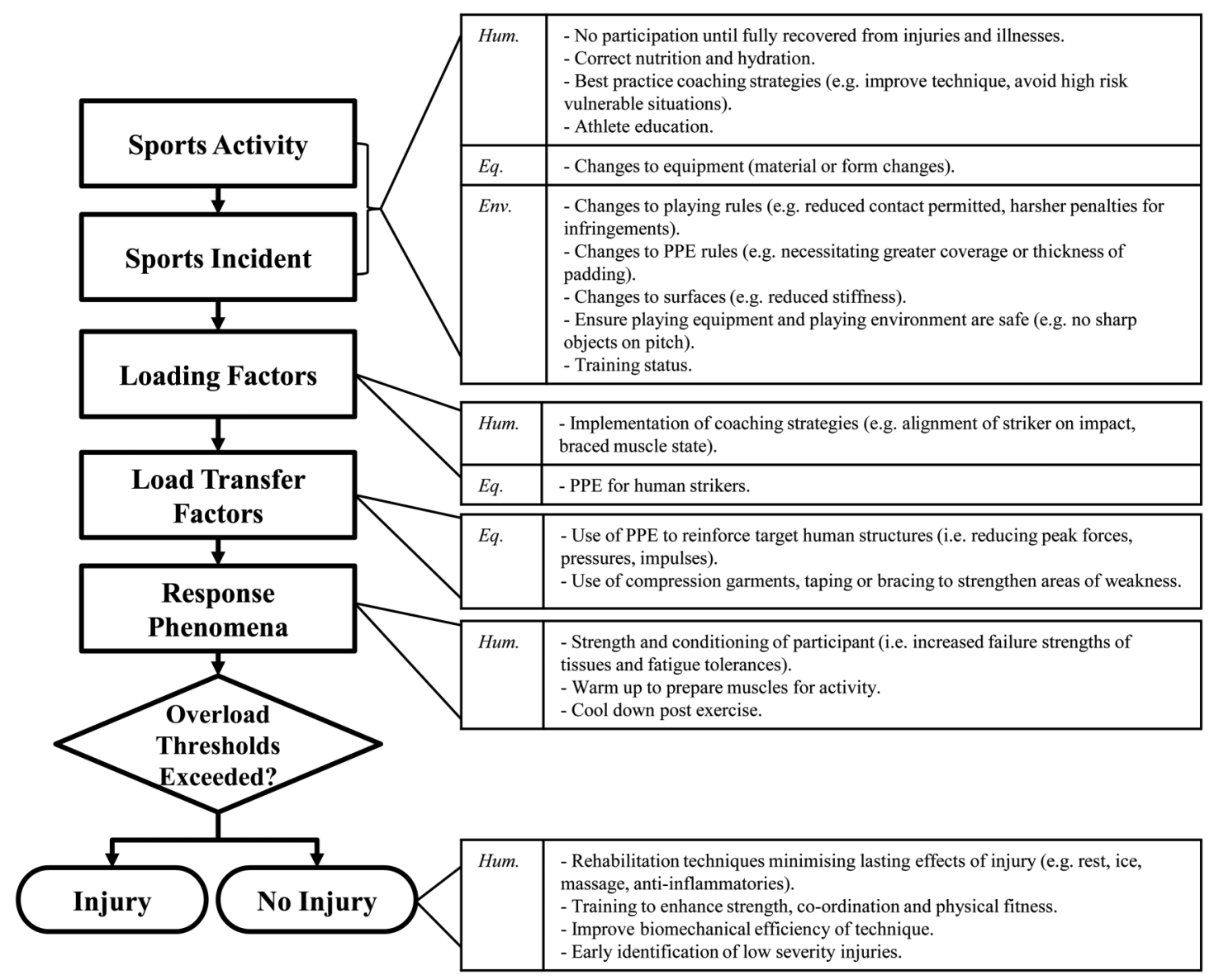

Figure 5 Example usage of the injury intervention opportunities model (Hum., Human; Eq., Equipment; Env., Environment). 
presents a detailed description of factors relating to a specific injury event and uses elements of best practice from biomechanical dependency chains, multifactorial models and the Haddon matrix to make a more complete, accessible and useful structure to define and investigate injuries.

The model's usefulness and external validity have been demonstrated through the range of applications established. No previously defined model has presented a sufficiently detailed framework that promotes consideration of as many pertinent influencing factors and directly enables the user to further investigate the injury event. For example, in the biomechanical dependency chain, the 'accident' entity is too broad and does not consider the context in which the impact was sustained. This could significantly affect the equipment and environment considered in gameplay reconstructions. Similarly, the risk identification and accumulation model would fail to describe, in sufficient detail, the mechanical loading phenomena which could prevent proper consideration of intervention opportunities (eg, appropriateness of personal protective equipment types).

An additional application of the model, not explicitly documented, is as a product design specification development aid for human surrogate development. The model can be applied as a framework to systematically identify important factors relating to a specific impact event and inform various elements of surrogate design and experimentation (eg, material selection, impact conditions and experimental design). This has been practically demonstrated in the development of a superior sports impact surrogate for personal protective equipment testing. ${ }^{32}$

\section{Limitations}

The models primary usage is in acute impact injuries and does not readily apply to chronic overuse injuries (eg, stress fractures) given the longitudinal and often recursive nature of the injury aetiology. A linear description of injuries is presented considering all pertinent epidemiological factors at the particular moment prior to the inciting event. Consequently, the model is not designed to address dynamic risk factors and a changing set of human injury tolerances and predispositions. ${ }^{7}$ In spite of this, through careful and accurate isolation of the factors relating to a specific event at a particular instant in time, these shortcomings can be overcome. For example, for a running foot injury, the model could be used to identify the nature of biomechanical loading at the instant prior to the injury as well as the ground surface conditions, equipment used (running shoes) and previous medical conditions.

Given the complexity of the model it may also be difficult to quantitatively assess all factors that are important to interpret the model in a truly useful form (eg, exact human tissue properties). These issues with classification are expected and the experimental protocol has been developed to address the paucity of information in certain sections.

A related drawback concerning the completeness of the model is that it can be time consuming to fully define an injury and may require additional research to generate useful information. However, the beneficial effects of a comprehensive description are believed to be worthwhile, this has been reflected by a statement by Bahr \& Krosshaug $^{6}$ who suggested that "a precise description of the inciting event is a key component to understanding the causes of a particular injury in a given sport" and advise that it is necessary to expand the typical biomechanical approach to fully describe the inciting event.

\section{SUMMARY}

The presented DCS model provides an improved structure to consider factors pertaining to a specific impact-injury event. A detailed series of influencing factors have been classified and can be used as a checklist to provide a comprehensive description of a particular injury. Useful applications for the model in: scenario definition, experimental protocol development and intervention opportunities have been outlined and offer practical guidance on methods to better understand and prevent injury.

Contributors TP and SM were responsible for initial conceptual model and review of applications. $\mathrm{BH}$ and $\mathrm{RB}$ were responsible for contributions to the applications of the model and proof reading and revisions of the manuscript.

Competing interests None declared.

Provenance and peer review Not commissioned; externally peer reviewed.

Open Access This is an Open Access article distributed in accordance with the Creative Commons Attribution Non Commercial (CC BY-NC 4.0) license, which permits others to distribute, remix, adapt, build upon this work noncommercially, and license their derivative works on different terms, provided the original work is properly cited and the use is non-commercial. See: http:// creativecommons.org/licenses/by-nc/4.0/

\section{REFERENCES}

1. Schmikli SL, Backx FJ, Kemler HJ, et al. National survey on sports injuries in the Netherlands: target populations for sports injury prevention programs. Clin J Sport Med 2009;19:101-6.

2. Merkle AC, Carneal CM, Wing ID, et al. Biomechanics and injury mitigation systems program: an overview of human models for assessing injury risk in blast, ballistic, and transportation impact scenarios. Johns Hopkins APL Tech Dig 2013;31:286-95.

3. Hagel B, Meeuwisse W. Risk compensation: a "side effect" of sport injury prevention? Clin J Sport Med 2004;14:193-6.

4. Meeuwisse W. Predictability of sports injuries. What is the epidemiological evidence? Sports Med 1991;12:8-15.

5. Meeuwisse WH. Assessing causation in sport injury: a mutifactorial model. Clin J Sport Med 1994;4:166-70.

6. Bahr R, Krosshaug T. Understanding injury mechanisms: a key component of preventing injuries in sport. $\mathrm{Br} J$ Sports Med 2005;39:324-9.

7. Meeuwisse WH, Tyreman $\mathrm{H}$, Hagel B, et al. A dynamic model of etiology in sport injury: the recursive nature of risk and causation. Clin J Sport Med 2007;17:215-19.

8. Gissane C, White J, Kerr K, et al. An operational model to investigate contact sports injuries. Med Sci Sports Exerc 2001;33:1999-2003.

9. Wismans J, Janssen EG, Beusenberg M, et al. Injury Biomechanics (4J610). 2000;w5-pp3-4.3. 
10. Mclntosh A. Risk compensation, motivation, injuries, and biomechanics in competitive sport. Br J Sports Med 2005;39:2-3.

11. Haddon WJ. Options for the prevention of motor vehicle crash injury. Isr J Med Sci 1980;16:45-65.

12. Bahr R, Mæhlum S. General injury prevention measures. Hong Kong: Human Kinetics, 2004.

13. Crandall JR, Kent R. A systems approach to sports injuries: turf toe and Lisfranc injuries in American Football. 2011. http://www.ircobi. org/downloads/2011-lecture-crandall.pdf 9th June 2012.

14. Verhagen E, van Mechelen W. The pragmatic approach. Sports injury research. New York: Oxford University Press, 2010:143-4.

15. van Mechelen W, Hlobil H, Kemper HC. Incidence, severity, aetiology and prevention of sports injuries. Sports Med 1992;14:82-99.

16. Nicholl JP, Coleman P, Williams BT. The epidemiology of sports and exercise related injury in the United Kingdom. Br J Sports Med 1995;29:232-8.

17. Steffen K, Andersen TE, Krosshaug T, et al. ECSS Position statement 2009: prevention of acute sports injuries. Eur J Sport Sci 2010;10:223-36.

18. Orchard J, Rae K, Brooks J, et al. Revision, uptake and coding issues related to the open access Orchard Sports Injury Classification System (OSICS) versions 8, 9 and 10.1. Open Access J Sports Med 2010;1:207-14.

19. Donnelly BR, Roberts D. Comparison of cadaver and hybrid III dummy response to axial impacts of the femur. SAE Paper No. 872204. 1987.

20. Ankrah S, Mills N. Analysis of ankle protection in Association football. Sports Eng 2004;7:41-52.

21. Halkon B, Webster J, Mitchell S, et al. Development of a test methodology for the assessment of human impacts in sport. 9th Conference of the International Sports Engineering Association (ISEA). 9-13 July 2012; Lowell, MA, USA: Elsevier, 2012.

22. Halkon BJ, Mitchell SR, Payne T, et al. Biomechanical measurements of human impacts in basketball. The 2014
Conference of the International Sports Engineering Association. Vol 72, 2014:214-19.

23. Payne T, Mitchell S, Bibb R, et al. The evaluation of new multi-material human soft tissue simulants for sports impact surrogates. J Mech Behav Biomed Mater 2015;41:336-56.

24. Hendricks S, Lambert MI, Brown JC, et al. An evidence-driven approach to scrum law modifications in amateur rugby played in South Africa. Br J Sports Med 2014;48:1115-19.

25. Silver J, Stewart D. The prevention of spinal injuries in rugby football. Spinal Cord 1994;32:442-53.

26. Quarrie KL, Gianotti SM, Hopkins WG, et al. Effect of nationwide injury prevention programme on serious spinal injuries in New Zealand rugby union: ecological study. BMJ 2007;334: 1150-3.

27. Donaldson A, Poulos RG. Planning the diffusion of a neck-injury prevention programme among community rugby union coaches. Br J Sports Med 2014;48:151-9.

28. Milburn PD. The kinetics of rugby union scrummaging. J Sports Sci 1990;8:47-60

29. Preatoni E, Stokes K, England M, et al. Forces generated in rugby union machine scrummaging at various playing levels. 2012 IRCOBI Conference Proceedings-International Research Council on the Biomechanics of Injury. 12-14 September 2012.

30. Cazzola D, Preatoni E, Stokes KA, et al. A modified prebind engagement process reduces biomechanical loading on front row players during scrummaging: a cross-sectional study of 11 elite teams. Br J Sports Med 2015;49:541-6.

31. Naish R, Burnett A, Burrows $S$, et al. Can a specific neck strengthening program decrease cervical spine injuries in a men's professional rugby union team? A retrospective analysis. J Sports Sci Med 2013;12:542-50.

32. Payne T, Mitchell SR, Halkon BJ, et al. Development of a synthetic human thigh impact surrogate for sports personal protective equipment testing. Proc Inst Mech Eng [P] 2015; [in press]. 